\title{
On A Posteriori Error Estimates
}

\author{
By George Miel
}

Abstract. Consider a sequence $\left\{x_{n}\right\}_{n=0}^{\infty}$ in a normed space $X$ converging to some $x^{*} \in X$. It is shown that the sequence satisfies a condition of the type

$$
\left\|x^{*}-x_{n}\right\| \leqslant \alpha\left\|x_{n}-x_{n-1}\right\|
$$

for some constant $\alpha$ and every $n \geqslant 1$, if the associated null sequence $\left\{e_{n}\right\}_{n=0}^{\infty}, e_{n}$ $=x^{*}-x_{n}$, is uniformly decreasing in norm or if it is alternating with respect to any ordering whose cone of positive elements is acute.

1. Introduction. A frequent situation deals with the approximation of an element $x^{*}$ of a metric space $X$ by a sequence $\left\{x_{n}\right\}_{n=0}^{\infty}$ in $X$ known to converge to $x^{*}$. During actual computations, the problem of course is to stop at an element $x_{n}$ close enough to the desired limit $x^{*}$. Letting $d$ denote the metric, a common criterion consists of stopping when $d\left(x_{n}, x_{n-1}\right)$ is within a given tolerance, thus hoping that $d\left(x^{*}, x_{n}\right)$ is reasonably small. This heuristic rule provides an actual error bound (assuming no roundoff errors) if the sequence is known to satisfy the condition

$$
d\left(x^{*}, x_{n}\right) \leqslant \alpha d\left(x_{n}, x_{n-1}\right),
$$

for some constant $\alpha>0$ and every $n \geqslant 1$. We call this condition a stopping inequality with constant $\alpha$.

Such an inequality holds for the sequence of successive iterates of a contractive mapping. Leibnitz' estimate of the remainder of an alternating series yields a stopping inequality for the sequence of partial sums. Knopp [12, Section 34] essentially used stopping inequalities to estimate the remainder of certain series; see also [15, Section 1.2]. Applications to continued fractions have been studied in a series of papers: Blanch [2], Henrici and Pfluger [8], Merkes [14], Gragg [6] , [7], Jefferson [10], Jones and Thron [11]. Ostrowski [18] applied the notion to the iterative solution of linear equations. Studies of a general nature were given by Varol [23] and Ostrowski [16] , [17, Appendix Q]. Rowland and Varol [20] showed that a sequence of compound Simpson quadrature rules, in which the number of panels is successively doubled, satisfies a stopping inequality for functions whose fourth derivative does not change sign. This result was extended to an arbitrary Newton-Cotes rule by Rowland and Miel [19].

Existing results on stopping inequalities are diverse and scattered. For this reason, this work adopts a general point of view, thus hoping to uncover some unifying notions.

Received March 29, 1976.

AMS (MOS) subject classifications (1970). Primary $65 \mathrm{~J} 05$.

Key words and phrases. A posteriori error estimates.

Copyright $\odot$ 1977, American Mathematical Society 
Our main result may be stated loosely as follows. A stopping inequality is readily available for a convergent sequence in a normed space provided that its associated sequence of errors is uniformly decreasing in norm (i.e., geometrically convergent) or if successive errors are on opposite sides of zero. The first condition is of a purely metric nature and is already known, Varol [20, p. 4] and Ostrowski [16]. The second condition can be made precise by order-theoretic considerations, namely, by requiring that the sequence of errors be alternating with respect to any ordering having the property that the cone of positive elements is not too wide. If the space has an inner product, then the width of the cone can be measured by a cosine. In this case, if the sequence of errors satisfies both the metric and the order conditions, then the resulting stopping inequality is sharper than the one that results from either single condition.

The above ideas are developed in Section 2. The next section gives selected examples and applications. Finally, Section 4 deals with the following problem: given linear mappings $x^{*}, x_{n}: X \rightarrow Y, n \geqslant 0$, such that $\lim x_{n}(f)=x^{*}(f)$ for every $f$, find constants $\alpha$ and subsets $S(\alpha)$ of $X$ such that

$$
\left\|x^{*}(f)-x_{n}(f)\right\| \leqslant \alpha\left\|x_{n}(f)-x_{n-1}(f)\right\|
$$

for every $f \in S(\alpha)$. The notions of Section 2 are applicable. It turns out that if $X$ and $Y$ are ordered, and if certain conditions are met, then the double-cone of positive and negative elements of $X$ is contained in some $S(\alpha)$.

Our notation is as follows. A metric space is denoted by a pair $(X, d)$ where $d$ is the metric. Only linear spaces over the real field $\mathbf{R}$ are considered in the sequel. We will expediently assume completeness, but it is clear that some of the results hold for a sequence in an incomplete space provided that it converges to an element of the space. We will use the standard symbols $\mathbf{R}^{n}, l^{p}, L^{p}[a, b],\|\cdot\|_{p}$ for the classical spaces and their norms.

We recall some order-theoretic notions, [9]. An ordering for a linear space $X$ is a reflexive and transitive relation $\leqslant$ such that

(i) if $x \leqslant y$, then $x+z \leqslant y+z$ for every $z \in X$, and

(ii) if $x \leqslant y$, then $\lambda x \leqslant \lambda y$ for every scalar $\lambda \geqslant 0$.

If $a \leqslant b$, then an order-interval is $[a, b]=\{x \mid a \leqslant x \leqslant b\}$. A set $A$ is order-convex if and only if it contains every order-interval $[a, b]$ with $a, b \in A$ and $a \leqslant b$.

A cone in a linear space $X$ is a nonvoid subset $P$ of $X$ such that $P+P \subset P$ and $\lambda P \subset P$ for every $\lambda \geqslant 0$. Some authors, for example Jameson [9], use the term wedge. The trivial cone is the singleton $\{0\}$. The cone associated to a given ordering $\leqslant$ is $P=$ $\{x \mid x \geqslant 0\}$. On the other hand, any given cone induces an ordering defined by: $x \leqslant y$ if and only if $y-x \in P$. We will denote an ordered linear space by $(X, P)$ where $P$ is the cone of positive elements. An ordered Banach space is denoted by $(X, P,\|\cdot\|)$ and an ordered Hilbert space by $(X, P,\langle\cdot, \cdot\rangle)$ where $\|\cdot\|$ and $\langle\cdot, \cdot\rangle$ are respectively the norm and inner product.

2. Development of the Theory. In this section, we derive fundamental conditions which imply stopping inequalities for sequences in metric and ordered normed spaces. 
TheOREM 2.1. Let $(X, d)$ be a metric space; suppose that the sequence $\left\{x_{n}\right\}_{n=0}^{\infty}$ in $X$ converges to some $x^{*} \in X$; let $\beta \in(0,1)$.

(i) If $d\left(x^{*}, x_{n}\right) \leqslant \beta d\left(x^{*}, x_{n-1}\right)$, then $d\left(x^{*}, x_{n}\right) \leqslant \alpha d\left(x_{n}, x_{n-1}\right)$ where $\alpha=$ $\beta(1-\beta)^{-1}$.

(ii) For any $\alpha>0$ there is a subsequence $\left\{x_{n_{p}}\right\}_{p=0}^{\infty}$ such that $d\left(x^{*}, x_{n_{p}}\right) \leqslant$ $\alpha d\left(x_{n_{p}}, x_{n_{p-1}}\right), p \geqslant 1$.

Proof. For (i), see [23, p. 4] or [16]. To prove (ii), suppose that $n_{0}=0, n_{1}$, $\ldots, n_{p-1}$ have been chosen. Since $\lim d\left(x^{*}, x_{n}\right)=0$, we can choose $n_{p}>n_{p-1}$ such that

$$
d\left(x^{*}, x_{n_{p}}\right) \leqslant \beta d\left(x^{*}, x_{n_{p-1}}\right), \quad \beta=\frac{\alpha}{\alpha+1} .
$$

The result follows from (i).

An important application of (i) occurs when the sequence $\left\{x_{n}\right\}$ consists of the iterates of a contractive mapping.

Next consider an ordered Banach space $(X, P,\|\cdot\|)$. A sequence $\left\{e_{n}\right\}_{n=0}^{\infty} \subset X$ is a null sequence if lim $\left\|e_{n}\right\|=0$. It is alternating if $(-1)^{n} \sigma e_{n} \in P$ where $\sigma$ is either 1 or -1 . Note that a sequence $\left\{x_{n}\right\} \subset X$, converging to an element $x^{*}$, satisfies the stopping inequality

$$
\left\|x^{*}-x_{n}\right\| \leqslant \alpha\left\|x_{n}-x_{n-1}\right\|
$$

if and only if the corresponding null sequence $\left\{e_{n}\right\}, e_{n}=x^{*}-x_{n}$, satisfies the same stopping inequality.

LEMMA 2.2. Let $B$ denote the closed unit ball of an ordered Banach space $(X, P,\|\cdot\|)$. Consider the following statement:

(i) $B$ is order-convex.

(ii) $\|x\| \leqslant\|x+y\|$ for every $x, y \in P$.

(iii) $\|x\| \leqslant\|z\|$ whenever $0 \leqslant x \leqslant z$.

Then (i) implies (ii), and (ii) is equivalent to (iii).

Proof. (i) $\Rightarrow$ (ii): Take nonzero elements $x, y \in P$. We have that $P \cap(-P)=$ $\{0\}$ and so $\|x+y\|=a>0$. Hence $0 \leqslant x / a \leqslant(x+y) / a$. But the order-interval [0, $(x+y) / a]$ is in $B$. Thus $x / a \in B$, which implies that $\|x / a\| \leqslant 1=\|(x+y) / a\|$.

(ii) $\Rightarrow$ (iii): Suppose that $0 \leqslant x \leqslant z$; then $x, y=z-x \in P$ and so $\|x\| \leqslant\|z\|$.

(iii) $\Rightarrow$ (ii): If $x, y \in P$, then $0 \leqslant x \leqslant x+y$. Hence $\|x\| \leqslant\|x+y\|$.

Definition 2.3. We say that an ordered Banach space $(X, P,\|\cdot\|)$ has an acute cone if condition (ii) or (iii) of Lemma 2.2 holds.

The term acute in the above definition is motivated by the interpretation of this property in terms of angles for inner product spaces. The lemma states that if a space has an order-convex unit ball then it has an acute cone of positive elements. The converse is not true as shown in Example 3.2 in the next section. However, we note that if a cone is acute then it is normal and so the space is then locally order-convex, $[9$, Section 3.2].

THEOREM 2.4. Let $(X, P,\|\cdot\|)$ be an ordered Banach space with an acute cone $P$. Any alternating null sequence $\left\{e_{n}\right\}_{n=0}^{\infty}$ in this space satisfies the stopping inequality $\left\|e_{n}\right\| \leqslant\left\|e_{n}-e_{n-1}\right\|, n \geqslant 1$. 
Proof. Either $e_{n},-e_{n-1} \in P$ or $-e_{n}, e_{n-1} \in P$. In either case, (ii) of Lemma 2.2 yields the result.

The following lemma characterizes an acute cone in an ordered Hilbert space.

Lemma 2.5. An ordered Hilbert space $(X, P,\langle\cdot, \cdot\rangle)$ has an acute cone if and only if $\langle x, y\rangle \geqslant 0$ for every $x, y \in P$.

Proof. We use the identity (law of cosines):

$$
\|x+y\|^{2}=\|x\|^{2}+\|y\|^{2}+2\langle x, y\rangle .
$$

The sufficiency is clear. To show necessity, suppose that $\langle x, y\rangle\langle 0$ for some $x, y$ in $P$. Choose $z=\lambda y$ where $\lambda>-\|x\|^{2} / 2\langle x, y\rangle$. Then $z \in P$ and

$$
\begin{gathered}
\langle x, y\rangle=\lambda\langle x, y\rangle\left\langle-1 / 2\|x\|^{2},\right. \\
\|z\|^{2}>\|x\|^{2}+\|z\|^{2}+2\langle x, z\rangle=\|x+z\|^{2},
\end{gathered}
$$

which implies that the cone is not acute.

Consider an ordered Hilbert space $(X, P,\langle\cdot, \cdot\rangle)$ with a nontrivial cone $P$; we define the cosine of $P$ by

$$
\gamma=\inf \{\langle x, y\rangle \mid x, y \in P \text { and }\|x\|=\|y\|=1\} .
$$

Observe that the space has an acute cone if and only if $\gamma \in[0,1]$, namely, if the angle is at most $\pi / 2$. In the following lemma, the backward difference $\nabla e_{n}$ is defined as usual by $\nabla e_{n}=e_{n}-e_{n-1}$.

LEMMA 2.6. Let $\left\{e_{n}\right\}$ be a sequence in an inner product space and let $\alpha>0$. Then $\left\|e_{n}\right\| \leqslant \alpha\left\|\nabla e_{n}\right\|$ if and only if $\left\langle\alpha \nabla e_{n}+e_{n}, \alpha \nabla e_{n}-e_{n}\right\rangle \geqslant 0$.

Proof. Expand the norms in the stopping inequality in terms of inner products.

In addition to Theorems 2.1 and 2.4 , the following results hold in ordered Hilbert spaces.

THEOREM 2.7. Let $\left\{e_{n}\right\}$ be a null sequence in an ordered Hilbert space $(X, P$, $\langle\cdot, \cdot\rangle)$ with a nontrivial acute cone $P$. Let $\beta$ be a constant in $(0,1)$ and let $\gamma$ be the cosine of $P$.

(i) $\left\|e_{n}\right\| \leqslant\left\|e_{n}-e_{n-1}\right\|$ if and only if $\left\langle e_{n-1}, e_{n}\right\rangle \leqslant 1 / 2\left\|e_{n-1}\right\|^{2}$.

(ii) If $\left\{e_{n}\right\}$ is contained either in $P$ or in $-P,\left\|e_{n}\right\| \leqslant\left\|e_{n}-e_{n-1}\right\|$, and $\gamma \geqslant 1 / 2$, then the sequence $\left\{\left\|e_{n}\right\|\right\}$ is monotone decreasing.

(iii) If $\left\|e_{n}\right\| \leqslant \beta\left\|e_{n-1}\right\|, n \geqslant 1$, and $\left\{e_{n}\right\}$ is alternating, then $\left\|e_{n}\right\| \leqslant \alpha^{\prime}\left\|e_{n}-e_{n-1}\right\|$, $n \geqslant 1$, where $\alpha^{\prime}=\beta\left(\beta^{2}+2 \beta \gamma+1\right)^{-1 / 2}$.

Proof. If $\alpha=1$, then $\left\langle\alpha \nabla e_{n}+e_{n}, \alpha \nabla e_{n}-e_{n}\right\rangle=-2\left\langle e_{n}, e_{n-1}\right\rangle+\left\|e_{n-1}\right\|^{2}$, and thus, (i) follows from Lemma 2.6.

To prove (ii), we use (i):

$$
\begin{gathered}
\left\langle e_{n-1}, e_{n}\right\rangle \leqslant 1 / 2\left\|e_{n-1}\right\|^{2}, \quad\left\langle\frac{e_{n-1}}{\left\|e_{n-1}\right\|}, \frac{e_{n}}{\left\|e_{n}\right\|}\right\rangle \leqslant \frac{1}{2} \frac{\left\|e_{n-1}\right\|}{\left\|e_{n}\right\|}, \\
\gamma \leqslant \frac{1}{2} \frac{\left\|e_{n-1}\right\|}{\left\|e_{n}\right\|}, \quad\left\|e_{n}\right\| \leqslant\left\|e_{n-1}\right\| .
\end{gathered}
$$


Finally, to prove (iii), assume without loss of generality that $-e_{n}, e_{n-1} \in P$ and use the law of cosines:

$$
\begin{aligned}
\left\|e_{n}-e_{n-1}\right\|^{2} & =\left\|e_{n}\right\|^{2}+\left\|e_{n-1}\right\|^{2}-2\left\langle e_{n}, e_{n-1}\right\rangle \\
& =\left\|e_{n}\right\|^{2}+\left\|e_{n-1}\right\|^{2}+2\left\|e_{n}\right\|\left\|e_{n-1}\right\|\left\langle\frac{-e_{n}}{\left\|e_{n}\right\|}, \frac{e_{n-1}}{\left\|e_{n-1}\right\|}\right\rangle \\
& \geqslant\left\|e_{n}\right\|^{2}+\frac{1}{\beta^{2}}\left\|e_{n}\right\|^{2}+\frac{2 \gamma}{\beta}\left\|e_{n}\right\|^{2} .
\end{aligned}
$$

The last inequality yields the desired result.

Consider the functions

$$
\alpha(\beta)=\beta(1-\beta)^{-1}, \quad \alpha^{\prime}(\beta, \gamma)=\beta\left(\beta^{2}+2 \beta \gamma+1\right)^{-1 / 2},
$$

where $0<\beta<1$ and $0 \leqslant \gamma \leqslant 1$. An elementary analysis shows that

(1) $\alpha^{\prime}(\beta, \gamma)<\alpha(\beta)$ for every $\beta, \gamma$.

(2) $\alpha(\beta)$ is strictly increasing and unbounded; also, $\alpha \leqslant 1$ if and only if $\beta \leqslant 1 / 2$.

(3) $\alpha^{\prime}(\beta, \gamma)$ is strictly increasing in $\beta$, strictly decreasing in $\gamma$, and $0<\alpha^{\prime}(\beta, \gamma)<$ $(2 \gamma+2)^{-1 / 2} \leqslant \sqrt{2} / 2$.

Hence, given a null sequence which satisfies the condition of (iii) of Theorem 2.7, the stopping inequality with constant $\alpha^{\prime}$ is sharper than the inequalities with constant 1 of Theorem 2.4 and with constant $\alpha$ of Theorem 2.1. Note also that the latter inequality is sharper than the former only when $\beta<1 / 2$.

3. Examples and Applications. Recall that the usual ordering of a linear space of real-valued functions on a set $T$ is defined by: $x \leqslant y$ if and only if $x(t) \leqslant y(t)$ for every $t \in T$. The cone associated with the usual ordering will be denoted by $P$.

3.1. Real Numbers. We may consider $\mathbf{R}$ as a Hilbert space with inner product $\langle x, y\rangle=x y$ and the theory of Section 2 holds. For example, statement (i) of Theorem 2.7 becomes: $\left|e_{n}\right| \leqslant\left|e_{n}-e_{n-1}\right|$ if and only if $e_{n-1}=0$ or $e_{n} / e_{n-1} \leqslant 1 / 2$.

Define a function $t: \mathbf{R}^{3} \rightarrow \mathbf{R}$ by $t(\alpha, x, y)=\alpha^{2}(y-x)^{2}-y^{2}$. As in Lemma 2.6, we can easily show that a null sequence $\left\{e_{n}\right\}$ in $\mathbf{R}$ satisfies a stopping inequality with constant $\alpha$ if and only if $t\left(\alpha, e_{n-1}, e_{n}\right) \geqslant 0, n \geqslant 1$. For fixed $\alpha$, the condition $t(\alpha, x, y)$ $\geqslant 0$ defines a double-cone in $\mathbf{R}^{2}$ bounded by the two lines $\alpha x-(\alpha-1) y=0$ and $\alpha x$ $-(\alpha+1) y=0$. Thus a null sequence $\left\{e_{n}\right\} \subset \mathbf{R}$ satisfies a stopping inequality with constant $\alpha$ if and only if the sequence $\left\{\left(e_{n-1}, e_{n}\right)\right\} \subset \mathbf{R}^{2}$ is in that double-cone.

3.2. Sequence Spaces. The usual cone $P$ is clearly acute in each of the spaces $l^{q}, 1 \leqslant q \leqslant \infty$. On the other hand, the cone associated with the lexicographic ordering is not acute in any one of these spaces. To see this, verify that $x=(0,1)$ and $y=$ $(1 / 2,-1)$ violate condition (ii) of Lemma 2.2 .

Let $B_{q}$ denote the closed unit ball of the space $\left(\mathbf{R}^{2}, P,\|\cdot\|_{q}\right) ; q=1,2, \infty$. The balls $B_{2}, B_{\infty}$ are order-convex but $B_{1}$ is not. Indeed, if $a=\left(a_{1}, a_{2}\right), b=\left(b_{1}, b_{2}\right) \in$ $B_{q}$ and $a \leqslant b$ then $b \in(a+P) \cap B_{q}$. The order-interval $[a, b]=\left\{x \mid a_{i} \leqslant x_{i} \leqslant b_{i}\right\}$ is a rectangle always contained in $B_{2}$ and $B_{\infty}$ but not necessarily in $B_{1}$.

3.3. Function Spaces. The usual cone $P$ is acute in each space $L^{q}[a, b], 1 \leqslant q \leqslant \infty$. 
Choose a sequence $\left\{x_{n}\right\}$ in $P \cap L^{2}[a, b]$ such that $\left\{\left\|x_{n}\right\|_{2}\right\}$ is monotone decreasing. Let $\left\{\epsilon_{n}\right\}$ be a null sequence in $\mathbf{R}$ and define a null sequence of functions $\left\{e_{n}\right\}$ by $e_{n}(t)=\epsilon_{n} x_{n}(t)$. Let $n \geqslant 1,0<\beta<1, \alpha=\beta(1-\beta)^{-1}$, and $\alpha^{\prime}=\beta\left(\beta^{2}+1\right)^{-1 / 2}$. Then

(1) If $\left|\epsilon_{n}\right| \leqslant \beta\left|\epsilon_{n-1}\right|$, then $\left\|e_{n}\right\|_{2} \leqslant \alpha\left\|e_{n}-e_{n-1}\right\|_{2}$.

(2) If $\left\{\epsilon_{n}\right\}$ is alternating, then $\left\|e_{n}\right\|_{2} \leqslant\left\|e_{n}-e_{n-1}\right\|_{2}$.

(3) If $\left|\epsilon_{n}\right| \leqslant \beta\left|\epsilon_{n-1}\right|$ and $\left\{\epsilon_{n}\right\}$ is alternating, then $\left\|e_{n}\right\|_{2} \leqslant \alpha^{\prime}\left\|e_{n}-e_{n-1}\right\|_{2}$. These statements follow from Theorems 2.1, 2.4, and 2.7.

Next, we give an example of spaces whose cones are not acute. Let $m \geqslant 1$ and consider the spaces $\left(C^{m}[a, b], P_{m},\|\cdot\|\right)$ where

$$
P_{m}=\left\{x \mid x^{(m)}(t) \geqslant 0 \text { for all } t\right\}, \quad\|x\|=\sum_{i=0}^{m}\left\|x^{(i)}\right\|_{\infty} .
$$

Verify that the functions $x(t)=t$ and $y(t)=-1 / 2$ violate condition (ii) of Lemma 2.2.

3.4. Infinite Series. Given a series $\Sigma a_{n}, a_{n} \in \mathbf{R}$, consider the stopping inequality with constant $\alpha$ for the corresponding sequence of partial sums. This means that

$$
\left|\sum_{i=1}^{\infty} a_{n+i}\right| \leqslant \alpha\left|a_{n}\right| .
$$

It can easily be shown [15, Theorem 1.2.2] that if a series passes the ratio test, namely, $\left|a_{n+1}\right| a_{n} \mid \leqslant r<1$, then it satisfies a stopping inequality with $\alpha=r(1-r)^{-1}$. Knopp $[12$, Section 34] thus gets error bounds for the series calculation of roots and logarithms.

3.5. Continued Fractions. Consider a convergent simple continued fraction with positive elements. Let $\left\{e_{n}\right\}$ denote the corresponding null sequence of errors. Then $\left\{e_{n}\right\}$ is alternating and $\left\{\left|e_{n}\right|\right\}$ is strictly decreasing, [3, p. 409]. It follows that $\left|e_{n}\right|$ $\leqslant 1 / 2\left|e_{n}-e_{n-1}\right|$. This stopping inequality can be applied, for example, on the Shanks procedure for calculating logarithms [22]. For various applications of stopping inequalities to continued fractions, see the references given in the introduction.

3.6. Linear Equations. Ostrowski [18] used the metric condition that the errors be uniformly decreasing in norm to get a posteriori error bounds for the iterative solution of linear equations.

Collatz and Schröder [4, pp. 352-353] gave conditions under which a type of splitting of nonsingular matrix yields monotone iterations under the usual ordering of $\mathbf{R}^{n}$. This result was extended for the least-squares solution of rectangular systems and for general orderings by Berman and Plemmons [1, Theorem 4]. One can thus get a sequence $\left\{x_{n}\right\}$ converging to a solution $x^{*}$ and such that

$$
x_{0} \leqslant \cdots \leqslant x_{2 k} \leqslant \cdots \leqslant x^{*} \leqslant \cdots \leqslant x_{2 k+1} \leqslant \cdots \leqslant x_{1} .
$$

If the cone associated to the ordering is acute, then the stopping inequality holds with $\alpha=1$, since the errors are successively positive and negative.

4. Sequences of Linear Mappings. In what follows, we make the following assumptions: $(X, P)$ is an ordered linear space, $(Y, Q,\|\cdot\|)$ is an ordered Banach space whose cone $Q$ is acute, and $e_{n}: X \rightarrow Y, n \geqslant 0$, is a sequence of linear mappings such 
that $\lim \left\|e_{n}(x)\right\|=0$ for every $x \in X$. This section deals with the problem of finding constants $\alpha$ and elements $x \in X$ such that $\left\|e_{n}(x)\right\| \leqslant \alpha\left\|e_{n}(x)-e_{n-1}(x)\right\|$.

Recall that a mapping $L: X \rightarrow Y$ is said to be positive if $L(P) \subset Q$. In particular, if $L$ is linear then $L$ is positive provided that $L(x) \leqslant L(y)$ whenever $x \leqslant y$. For any $\alpha$ $\in \mathbf{R}$, define the set

$$
S(\alpha)=\left\{x \in X \mid\left\|e_{n}(x)\right\| \leqslant \alpha\left\|e_{n}(x)-e_{n-1}(x)\right\|, n \geqslant 1\right\} .
$$

Elementary properties are:

$S(\alpha)$ always contains 0 ,

$\lambda x \in S(\alpha)$ for every $x \in S(\alpha)$ and every $\lambda \in \mathbf{R}$,

$S\left(\alpha^{\prime}\right) \subset S(\alpha) \quad$ whenever $\alpha^{\prime} \leqslant \alpha$.

The following theorem gives conditions which imply that the double-cone $P \cup(-P)$ is contained in some $S(\alpha)$.

THEOREM 4.1. Let $\sigma$ be either 1 or -1 , let $\beta$ denote a constant in $(0,1)$, and let $n=0,1,2, \ldots$.

(i) If $(-1)^{n} \sigma e_{n}$ are positive, then $P \cup(-P) \subset S(1)$.

(ii) If $\sigma e_{n}$ and $\beta \sigma e_{n}-\sigma e_{n+1}$ are positive, then $P \cup(-P) \subset S(\alpha)$ where $\alpha=$ $\beta(1-\beta)^{-1}$.

(iii) Suppose that $Y$ is a Hilbert space whose cone $Q$ is nontrivial and has cosine $\gamma$. If $(-1)^{n} \sigma e_{n}$ are positive and $\left\|e_{n+1}(x)\right\| \leqslant \beta\left\|e_{n}(x)\right\|$ for every $x \in P$, then $P \cup(-P)$ $C S\left(\alpha^{\prime}\right)$ where $\alpha^{\prime}=\beta\left(\beta^{2}+2 \beta \gamma+1\right)^{-1 / 2}$.

Proof. If $(-1)^{n} \sigma e_{n}$ are positive and if $x \in P \cup(-P)$, then $\left\{e_{n}(x)\right\}$ is an alternating null sequence in $(Y, Q,\|\cdot\|)$. Thus, Theorem 2.4 implies that $x \in S(1)$. This proves (i).

For (ii), if $x \in P \cup(-P)$, then $\sigma^{\prime} x \in P$ where $\sigma^{\prime}$ is 1 or -1 . Consequently, $\sigma e_{n}\left(\sigma^{\prime} x\right)$ and $\beta \sigma e_{n}\left(\sigma^{\prime} x\right)-\sigma e_{n+1}\left(\sigma^{\prime} x\right)$ are in $Q$. Hence

$$
0 \leqslant \sigma e_{n+1}\left(\sigma^{\prime} x\right) \leqslant \beta \sigma e_{n}\left(\sigma^{\prime} x\right) .
$$

Since the cone $Q$ is acute, condition (iii) of Lemma 2.2 holds. It follows that

$$
\left\|e_{n+1}(x)\right\| \leqslant \beta\left\|e_{n}(x)\right\| .
$$

Thus (i) of Theorem 2.1 implies that $x \in S(\alpha), \alpha=\beta(1-\beta)^{-1}$.

To prove (iii), note that if $x \in P \cup(-P)$ then $\left\{e_{n}(x)\right\}$ is an alternating null sequence and $\left\|e_{n+1}(x)\right\| \leqslant \beta\left\|e_{n}(x)\right\|$. The result follows from (iii) of Theorem 2.7.

In the following theorem, we consider two ordered Banach spaces $\left(X_{i}, P_{i},\|\cdot\|_{i}\right)$, a sequence of continuous linear functionals $e_{n}: X_{1} \rightarrow Y \equiv R, n \geqslant 0$, such that $\lim e_{n}(x)$ $=0$ for every $x$, and subsets $S(\alpha)$ of the space $X_{1}$. We recall some definitions [13, Section 4.3]. The dual $X_{i}^{*}$ of $X_{i}$ is the Banach space of all bounded linear functionals $\phi: X_{i} \rightarrow \mathbf{R}$, with the norm

$$
\|\phi\|=\sup _{\|x\|_{i} \leqslant 1}|\phi(x)|
$$


The adjoint of a bounded linear map $t: X_{1} \rightarrow X_{2}$ is the map $t^{*}: X_{2}^{*} \rightarrow X_{1}^{*}$ defined by $t^{*}(\phi)(x)=\phi \circ t(x)$. The null space of $t$ is $N(t)=\{x \mid t(x)=0\}$.

Consider a decomposition $e_{n}=u_{n} \circ t$ as shown in the following diagram:

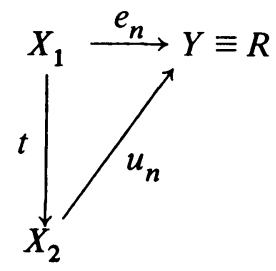

Sard's Theorem [21] yields such decompositions. Peano's Theorem [5, p. 70] is the special case when $e_{n}$ is a certain type of error functional annihilating polynomials of some degree. The next theorem gives conditions on such decompositions so that $P_{1} \cup$ $\left(-P_{1}\right)$ is contained in some $S(\alpha)$.

THEOREM 4.2. Let $t: X_{1} \rightarrow X_{2}$ be a continuous, linear, surjective, and positive mapping such that $N(t) \subset \bigcap_{n \geqslant 0} N\left(e_{n}\right)$. Define a sequence $\left\{u_{n}\right\}_{n=0}^{\infty} \subset X_{2}^{*}$ by $u_{n}=$ $\left(t^{*}\right)^{-1}\left(e_{n}\right)$. Let $\sigma$ be either 1 or -1 , let $\beta$ be a constant in $(0,1)$, and let $n=1,2, \ldots$.

(i) If $(-1)^{n} \sigma u_{n}$ are positive, then $P_{1} \cup\left(-P_{1}\right) \subset S(1)$.

(ii) If $\sigma u_{n}$ and $\beta \sigma u_{n}-\sigma u_{n+1}$ are positive, then $P_{1} \cup\left(-P_{1}\right) \subset S(\alpha)$ where $\alpha=$ $\beta(1-\beta)^{-1}$.

(iii) If $(-1)^{n} \sigma u_{n}$ are positive and $\left|u_{n+1}(\phi)\right| \leqslant \beta\left|u_{n}(\phi)\right|$ for every $\phi \in P_{2}$, then $P_{1} \cup\left(-P_{1}\right) \subset S\left(\alpha^{\prime}\right)$ where $\alpha^{\prime}=\beta(1+\beta)^{-1}$.

Proof. A special case of Sard's Theorem [13, p. 253] implies that $e_{n}=u_{n} \circ t$.

Define

$$
T(\alpha)=\left\{y \in X_{2}|| u_{n}(y)|\leqslant \alpha| u_{n}(y)-u_{n-1}(y) \mid, n \geqslant 1\right\} .
$$

Theorem 4.1 applied on $\left\{u_{n}\right\}$ yields

(i) $P_{2} \cup\left(-P_{2}\right) \subset T(1)$,

(ii) $P_{2} \cup\left(-P_{2}\right) \subset T\left(\beta(1-\beta)^{-1}\right)$,

(iii) $P_{2} \cup\left(-P_{2}\right) \subset T\left(\beta(1+\beta)^{-1}\right)$.

In (iii), we used the fact that the usual cone in $\mathbf{R}$ has cosine $\gamma=1$. The positivity of $t$ implies that $y=t(x) \in P_{2} \cup\left(-P_{2}\right)$ for every $x \in P_{1} \cup\left(-P_{1}\right)$. Thus, if $P_{2} \cup\left(-P_{2}\right)$ $\subset T(\lambda)$ then $P_{1} \cup\left(-P_{1}\right) \subset S(\lambda)$. The desired conclusions follow.

Example 4.3. Let $N \geqslant 1$ be a fixed integer; let $d=N$ if $N$ is odd and $d=N+1$ if $N$ is even; let $x_{n}(f)$ denote the value of the compound $(N+1)$-point Newton-Cotes quadrature rule with $2^{n}$ panels applied on a function $f$; let $x^{*}(f)$ be the exact value of the desired definite integral. It can be shown that

$$
\left|x^{*}(f)-x_{n}(f)\right| \leqslant\left|x_{n}(f)-x_{n-1}(f)\right|, \quad n \geqslant 1,
$$

for any function $f$ which has a continuous $(d+1)$ th derivative of constant sign. We now relate this result to Theorem 4.2.

Consider the ordered Banach spaces

$$
X_{1} \equiv\left(C^{d+1}[a, b], P_{1},\|\cdot\|_{d+1}\right), \quad X_{2} \equiv\left(C[a, b], P_{2},\|\cdot\|_{0}\right),
$$


where

$$
\|f\|_{q}=\max _{0 \leqslant i \leqslant q}\left\|f^{(i)}\right\|_{\infty}
$$

$P_{2}$ is the cone associated with the usual ordering of $C[a, b]$,

$$
P_{1}=t^{-1}\left(P_{2}\right), \quad t: X_{1} \rightarrow X_{2}, \quad t(f)=f^{(d+1)} \text {. }
$$

Consider the error functionals $e_{n}: X_{1} \rightarrow R, e_{n}(f)=x^{*}(f)-x_{n}(f)$. Since each $e_{n}$ annihilates the polynomials of degree $d$, there is a decomposition

$$
e_{n}=u_{n} \circ t, \quad u_{n}(\phi)=\int_{a}^{b} \phi(t) K_{n}(t) d t,
$$

where $K_{n}(t)$ is the Peano kernel associated to $e_{n}$.

By choice of $P_{1}, t$ is positive. The functionals $\sigma u_{n}, \sigma=-1$, are positive because the kernels $K_{n}(t)$ are nonpositive. By (ii) of Theorem 4.2, if we can show that there is a constant $\beta \in(0,1)$ such that the mappings $\beta \sigma u_{n}-\sigma u_{n+1}$ are positive, then $P_{1} \cup$ $\left(-P_{1}\right) \subset S\left(\beta(1-\beta)^{-1}\right)$. However, it can be shown (the proof is involved [19]) that the inequality

$$
\sigma K_{n+1}(t) \leqslant \beta \sigma K_{n}(t), \quad t \in[a, b],
$$

holds with $\beta=1 / 2$. The pointwise inequality of the kernels implies the positivity of the functionals $1 / 2 \sigma u_{n}-\sigma u_{n+1}$. Consequently, $P_{1} \cup\left(-P_{1}\right) \subset S(1)$.

5. Conclusion. This paper adopted a general point of view in order to find unifying notions regarding stopping inequalities. It turns out that such an inequality holds for a null sequence in a normed space if it is uniformly decreasing in norm or if it is alternating with respect to some ordering whose cone is acute. This principle should perhaps be of interest in the investigation of new applications of a posteriori error estimates.

Acknowledgment. The author wishes to thank Professor John H. Rowland for his guidance during the preparation of this work.

Department of Mathematics

University of Wyoming

Laramie, Wyoming 82071

1. A. BERMAN \& R. J. PLEMMONS, "Cones and iterative methods for best least squares solutions of linear systems," SIAM J. Numer. Anal., v. 11, 1974, pp. 145-154. MR 50 \#1478.

2. G. BLANCH, "Numerical evaluation of continued fractions," SIAM Rev., v. 6, 1964, pp. 383-421. MR 30 \#1605.

3. G. CHRYSTAL, Algebra, Vol. 2, Adam and Black, Edinburgh, 1889; 6th ed., reprinted, Chelsea, New York, 1959. MR 22 \#12066.

4. L. COLLATZ, Functional Analysis and Numerical Mathematics, Springer-Verlag, Berlin, 1964; English transl., Academic Press, New York, 1966. MR 29 \#2931; 34 \#4961.

5. P. J. DAVIS, Interpolation and Approximation, Blaisdell, New York, 1963. MR 28 \#393.

6. W. B. GRAGG, "Truncation error bounds for $g$-fractions," Numer. Math., v. 11, 1968, pp. 370-379. MR 37 \#3742.

7. W. B. GRAGG, “Truncation error bounds for $\pi$-fractions," Bull. Amer. Math. Soc., v. 76, 1970, pp. $1091-1094$. MR 41 \#328.

8. P. HENRICI \& P. PFLUGER, "Truncation error estimates for Stielties fractions," Numer. Math., v. 9, 1966, pp. 120-138. MR 35 \#3856. 
9. G. JAMESON, Ordered Linear Spaces, Lecture Notes in Math., vol. 141, Springer-Verlag, Berlin and New York, 1970.

10. T. H. JEFFERSON, "Truncation error estimates for $T$-fractions," SIAM J. Numer. Anal., v. 6, 1969, pp. 359-364. MR 41 \#475.

11. W. B. JONES \& W. J. THRON, "A posteriori bounds for the truncation error of continued fractions," SIAM J. Numer. Anal., v. 8, 1971, pp. 693-705. MR 45 \#4602.

12. K. KNOPP, Theory and Application of Infinite Series, Blackie \& Son Ltd., London and Glasgow, 1928.

13. P.-J. LAURENT, Approximation et Optimisation, Hermann, Paris, 1972.

14. E. P. MERKES, "On truncation errors for continued fraction computations," SIAM J. Numer. Anal., v. 3, 1966, pp. 486-496. MR 34 \#2156.

15. G. MIEL, On A Posteriori Error Estimates, Dissertation, Dept. of Math., Univ. of Wyoming, Laramie, 1976.

16. A. M. OSTROWSKI, "Les estimations des erreurs a posteriori dans les procédés itératifs," C. R. Acad. Sci. Paris Sér. A, v. 275, 1972, pp. 275-278.

17. A. M. OSTROWSKI, Solution of Equations in Euclidean and Banach Spaces, 3rd ed., Academic Press, New York, 1973. MR 50 \#11760.

18. A. M. OSTROWSKI, "A posteriori error estimates in iterative procedures," SIAM J. Numer. Anal., v. 10, 1973, pp. 290-298. MR 49 \#4202.

19. J. H. ROWLAND \& G. MIEL, "Exit criteria for Newton-Cotes quadrature rules," SIAM J. Numer. Anal. (To appear.)

20. J. H. ROWLAND \& Y. L. VAROL, “Exit criteria for Simpson's compound rule," Math. Comp., v. 26, 1972, pp. 699-703. MR 49 \#6569.

21. A. SARD, "Integral representations of remainders," Duke Math. J., v. 15, 1948, pp. 333-345. MR 10, 197.

22. D. SHANKS, “A logarithm algorithm," MTAC, v. 8, 1954, pp. 60-64. MR 15, 830.

23. Y. L. VAROL, Exit Criteria for Some Numerical Algorithms, Dissertation, Dept. of Math., Univ. of Wyoming, Laramie, 1971. 